\title{
Dephasing of optically induced excitonic coherences in semiconductor heterostructures
}

\author{
D.G. Steel ${ }^{1}$, H. Wang ${ }^{2}$ \\ ${ }^{1}$ The Harrison M. Randall Laboratory of Physics, The University of Michigan, Ann Arbor, USA \\ ${ }^{2}$ The Physics Department, The University of Oregon, Eugene, USA \\ Received: 30 March 2000/Accepted: 2 September 2000/Published online: 12 October 2000 - (c) Springer-Verlag 2000
}

\begin{abstract}
Resonant optical excitation of a direct bandgap semiconductor below the band edge induces excitonic coherences. Experiments based on transient four-wave mixing or cw spectral-hole burning provide an excellent approach to eliminate inhomogeneous broadening and enable determination of the time scale and origin of the decay of the optically induced quantum coherence. Such measurements are of interest in basic physics since they reflect fundamental interactions between the exciton and the surrounding environment including, for example, lattice vibrations and interface fluctuations. They also relate to potential applications of these excitations such as in coherent control or quantum information processing.
\end{abstract}

PACS: 71.35.-y; 78.47.+p; 39.30.+w

Optical excitations near the bandedge of direct bandgap semiconductors and semiconductor heterostructures are dominated by the contributions from strong excitonic resonances that form just below the bandedge. In the absence of disorder leading to inhomogeneous broadening, the spectral linewidth of these resonances reflects the decay of optically induced quantum coherence due to dephasing. Understanding the physics of the dephasing process, which can be due to energy relaxation as well as pure dephasing, i.e., processes that lead to a loss of the coherence but with no changes in excitonic population, provides important information on interactions between the exciton and the surrounding environment including, for example, lattice vibrations and interface fluctuations. This information is not only of fundamental importance but also critical for many potential applications such as coherent control and quantum information processing proposed recently.

Dephasing represents the decay of the phase correlation between two states that are prepared as a coherent superposition state, for example $|\Psi\rangle=C_{1}|1\rangle+C_{2} \mathrm{e}^{\mathrm{i} \omega t}|2\rangle$ where $\hbar \omega=E_{2}-E_{1}$. Dephasing can simply arise from a decay of the probability amplitude of one of the states due to population relaxation such as spontaneous emission. Such energy relaxation processes can also be observed in the time evolution of the population of the relevant state $\propto\left|C_{i}\right|^{2}$, which is phase insensitive. Measurement of the dephasing rate, however, follows from probing an observable that is sensitive to the relative phase between $C_{1}$ and $C_{2}$. Of particular interest to the focus of this paper, is the dephasing rate associated with the dipole coherence $\propto C_{1} C_{2}^{*} \mathrm{e}^{-\mathrm{i} \omega t}+c c$. If the population decays at a rate, $\Gamma$, then the dipole coherence decays at a rate $\gamma=\Gamma / 2$. Processes that lead to loss of coherence between $C_{1}$ and $C_{2}$ (dipole or other coherence) without a decay of the amplitude of either $C_{1}$ or $C_{2}$ are referred to as pure dephasing and result in a total dephasing rate of $\gamma=\Gamma / 2+\gamma_{\mathrm{ph}}$ with $\gamma_{\mathrm{ph}}$ being the pure dephasing rate. Theories that have considered the complications of general dephasing processes for excitons at low temperature in semiconductors are in general more complex than those in simpler atomic type systems (see for example [1-5]).

In a homogeneously broaden system, one can either measure the line shape of the dipole transition, which will yield a Lorentzian corresponding to the Fourier transform of decay of the dipole coherence, or directly probe the decay by time resolving the emitted coherent radiation following an impulse resonant excitation. Figure 1 shows as an example the time resolved free polarization decay (FPD) from homogeneously broadened exciton resonances in a bulk thin film of GaAs [6]. In this study, the sample was slightly strained to lift the heavy-hole (hh) and light-hole (lh) degeneracy. The FPD shows a simple exponential decay of the dipole induced coherence with a series of dipole coherence beats superimposed on the decay representing the interference of the coherent radiation emitted from the hh and lh excitons.

In heterostructures such as quantum wells and quantum dots, the excitonic resonances as observed in photoluminescence (PL) or photoluminescence excitation (PLE) are broadened far beyond the fundamental linewidth from dephasing due to inhomogeneous broadening induced by interface disorder. Confinement of excitons by the potential barriers leads to a shift of the excitonic resonances from that expected in the ideal three dimensional case. Fluctuations in the well thickness, which are known to occur on various length scales, lead to spatially dependent shifts in the energy level struc- 


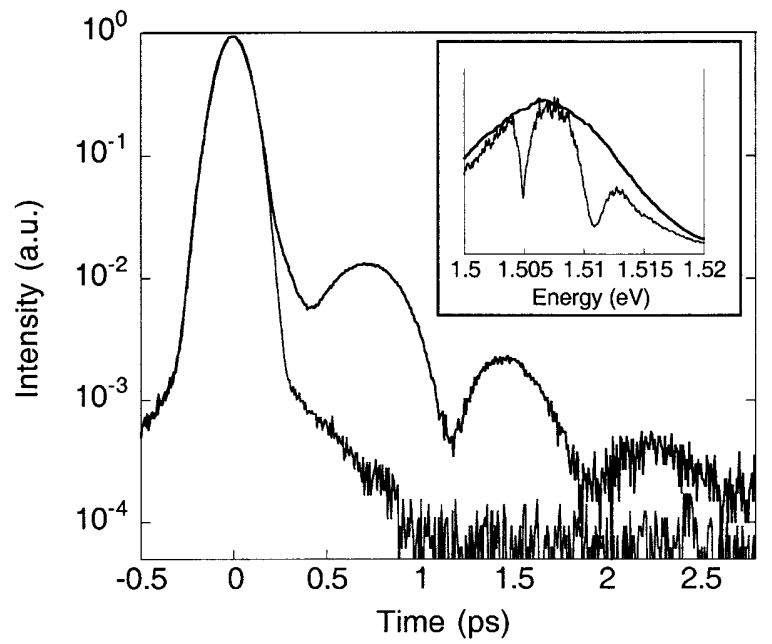

Fig. 1. Time resolved emission of the free polarization decay following excitation of the homogeneously broadened hh- and lh-exciton lines in a strained layer of bulk GaAs. Inset: frequency spectra of the co-polarized emission. The overall decay represents the loss of dipole induced coherence at the dephasing rate. The oscillations of dipole coherence beats between the heavy-hole and light-hole exciton

ture. A macroscopic average of these effects lead to significant inhomogeneous broadening of the resonance by typically a factor of 10 or more.

Two very different approaches can be used to obtain information on intrinsic dephasing processes in these inhomogeneously broadened systems. Near field linear [7] and nonlinear optical spectroscopy $[8,9]$ provide adequate spatial resolution to enable the study of single isolated excitons, thus eliminating effects of inhomogeneous broadening associated with an ensemble average. Coherent nonlinear optical spectroscopy in the far field based on third order nonlinear optical processes also provides similar information on dephasing as well as enabling, with relative ease, a more complete determination of various dephasing processes such as spectral and spatial diffusion and pure dephasing. It should be noted that measurements at very high spatial resolution [7] have recovered the narrow linewidth first reported using coherent nonlinear optical spectroscopy. In addition, these studies also show that in some cases, the dephasing process itself can be the origin of the nonlinear optical response, as discussed below.

Nonlinear optical spectroscopy based on the third order nonlinear optical response can be viewed as a four-wave mixing process and can be carried out in either the transient or frequency domain. The most common approach in use is transient four-wave mixing which, in the presence of inhomogeneous broadening, leads to the ordinary or stimulated photon echo, depending on geometry. This technique highlights short time scale relaxation phenomena but lacks spectral sensitivity. Four-wave mixing in the spectral domain is closely related to the older methodology of spectral hole burning. This approach tends to emphasize the relatively longer time scale phenomena but provides excellent spectral resolution and maximum intrinsic signal-to-noise since the duty cycle for the measurement is $100 \%$.

A significant challenge in these nonlinear optical measurements, particularly for the time domain measurements, is that in order to probe intrinsic dephasing phenomena, the ex-

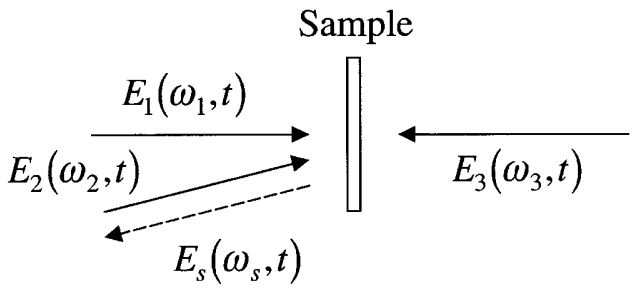

Fig. 2. Optical configuration for 3-beam FWM in the phase conjugate geometry

citation density must remain very low. This is because of the large scattering cross section for exciton-exciton and excitonelectron/scattering [10-13]. In the case of typical quantum wells, it is thus important to avoid exciting the continuum and to keep the exciton density well below $10^{9}$ excitons $/ \mathrm{cm}^{2}$. In most cases, this is readily obtained, but requires the use of low noise geometries, phase sensitive detection, and signal averaging. It is then further necessary to confirm that all measurements are truly density independent.

The lowest noise experimental approach with the greatest measurement capability is based on 3-beam four-wave mixing in the phase conjugate geometry, shown in Fig. 2. An excellent review of the physics and the details pertaining to the various measurements that can be made can be found for transient FWM excitation in $[14,15]$ and for $\mathrm{cw}$ FWM in $[16,17]$. The $E_{1}$ field is amplitude modulated allowing for phase sensitive detection of the signal. In some cases, it is important to amplitude-modulate at high frequency $(>100 \mathrm{MHz})$ to reduce the contribution to the signal from slow contributions to the nonlinear optical response, such as thermal effects, or in some cases even undesirable physical processes such as spectral diffusion [18]. For coherent transient excitation, the modern titanium-sapphire lasers provide excellent amplitude stability, but typically, their pulsewidth is too short resulting in a pulse bandwidth that excites multiple states and the continuum. For dephasing measurements, it is necessary to reduce the pulsewidth to $5-10 \mathrm{psec}$, though care is needed to ensure an adequate contrast in the pulse shape. Measurements presented in this paper were made, for the most part, with synchronously pumped modelocked dye lasers which have excellent contrast in their pulse shape. For the frequency domain measurements, it is necessary that the lasers be frequency locked and continuously tunable (e.g., the model 899-21 or 899-29 series from Coherent, Inc.) Diode lasers in principle are good, but do not yet have the necessary tunability needed for many experiments, and in some cases side band suppression may not be adequate.

In the following, we will examine in detail results from both high resolution $\mathrm{cw}$ and coherent transient nonlinear optical spectroscopic studies and show how these studies reveal fundamental processes contributing to dephasing. In addition, we also show that the role of disorder is extremely complex, resulting in significant qualitative changes in the physical behavior.

\section{CW spectral hole burning}

Because of the high resolution of frequency domain spectroscopy, spectral-hole-burning is an extremely informative especially since it can also directly reveal spectral diffusion 
processes. In these experiments, the fields shown in Fig. 2 are derived from frequency stabilized tunable lasers. Typically, $\omega_{2}-\omega_{1}=\delta$ and $\delta$ is adjusted to be smaller than energy relaxation time scales of interest. In the simplest case, the two fields come from the same laser and $\delta=0$. These fields are tuned to a given frequency within the inhomogeneous absorption profile. The signal is recorded as a function of $\omega_{3}$.

A simple example of the result is shown in Fig. 3 where data is obtained for a $2 \mathrm{meV}$ broad photoluminescence hhexciton line in a 60 period multiple quantum well [19]. The line shape is considerably more complex than that of a simple inhomogeneously broadened resonance [16] which would appear as a simple Lorentzian with FWHM of $4 \gamma$. Indeed, it is well known that $\mathrm{cw}$ measurements emphasize long time scale events. The low energy tail of this line shape is in fact due to spectral diffusion, i.e., the movement of excitons at energy $E$ to energy $E^{\prime}$. A measurement of the temperature dependence of the rate of migration shows that the mechanism of migration appears to be due to phonon assisted tunneling, though thermal activation may also contribute. At low temperatures, the phonon assisted migration model developed by Takagahara [2] shows that excitons resonantly excited are in a nonequilibrium state and can migrate to other sites by emitting or absorbing acoustic phonons. The migration is due to the overlap of the exciton wave function in different sites when the inter-site distance is small; the inter-site dipole-dipole interaction mediates the migration process when the inter-site distance is much greater than the localization length. The typical magnitude of participating phonon wave vectors is within a few times of the inverse of the localization length corresponding to phonon energies of order 0.01 to $0.1 \mathrm{meV}$. The theory further predicts a distinctive temperature dependence for the migration rate. At low temperatures, the dependence is described by $\exp \left(B T^{\alpha}\right)$. In this expression, $B$ is positive and independent of temperature but is expected to increase with the exciton energy and depends on details of interface roughness; $\alpha$ is estimated to be between 1.6 and 1.7. The predicted temperature dependence is quite different from that of variable range hopping used by Mott to interpret electronic conduction in the localized regime [20]. The difference has been attributed to the long-range nature of the inter-site interaction and the phonon emission process involved in the

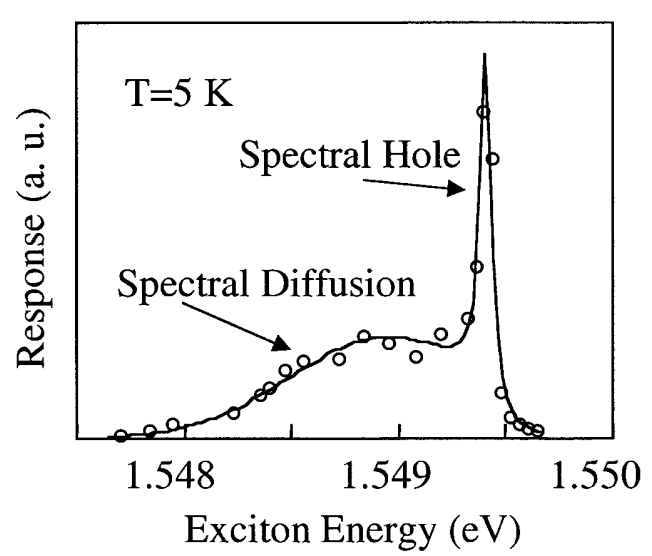

Fig. 3. The FWM spectral hole burning response with the two forward beams tuned $1.5 \mathrm{meV}$ below line center. The data shows the narrow spectral hole as well as the presence of excitation below line center due to spectral diffusion of excitons excited within the spectral hole migration of the localized exciton. The temperature dependence has been observed in transient hole burning experiments in an InGaAs/InP QW where all excitons are localized by alloy disorder [21]. Measurements of the relaxation rate in this system confirm this behavior at low temperature, and the dephasing is due to decay of exciton excited at the laser wavelength to another state.

From these measurements, it is hard to know if the width of the narrow line is due entirely to dephasing, or whether it has a component due to spectral diffusion, a problem usually associated with $\mathrm{cw}$ measurements which are known to emphasize long time scale events. Hence, time domain measurements are usually considered more advantageous in this regard. However, it is in fact straight forward to suppress long time scale events by setting a frequency difference between fields $E_{1}$ and $E_{2}$ that is comparable or larger than the relaxation time of the long lived components. This was required for studies of the exciton $g$-factor and showed that the narrow feature in Fig. 3 showed minimal broadening due to spectral diffusion [18]; i.e., the linewidth was due entirely to dephasing due to phonon assisted migration.

While exciton-phonon interactions clearly lead to spectral diffusion and dephasing, other extrinsic mechanisms leading to dephasing include scattering by incoherently generated electrons/holes and excitons. A comprehensive study of this behavior was made using transient self diffracted four-wave mixing [10]. The measurements showed that generation of free electrons is about an order of magnitude more effective at inducing dephasing than free excitons.

\section{Transient four wave mixing}

Given the rapid development of ultrafast laser technology and the prevalence of these systems in laboratories, coherent time domain measurements provide a relatively easy means to follow dephasing processes. An extensive review of the physics of this measurement is given in [14]. In an ideal homogeneously broadened system, the FWM interaction leads to emission of the coherent free polarization decay in the direction determined by phase matching (counter propagating with respect to $E_{2}$ in the phase conjugate geometry). However, if the system is inhomogeneously broadened, integration overall the radiators leads to destructive interference of the signal following the third pulse and no signal, initially. At the time $t_{2}-t_{1}$, following the third pulse, constructive interference occurs, producing a signal called the stimulated photon echo (or simply a photon echo in the case of two-beam self diffracted FWM) $[22,23]$ with an amplitude that exponentially decays according to $4 \gamma$ if the echo intensity is time integrated. In a time resolved experiment, with transformed limited pulses, the width of the echo is determined by the inhomogeneous width (assuming a simple Gaussian distribution). The first report of the simplest echo, a two beam self diffracted time resolved photon echo was presented in [24]. These measurements provided the first indication that the rephasing of the dipole coherences in an inhomogeneously broadened system needed to observe an echo (i.e., a signal that occurs at time $t_{2}-t_{1}$ after the second pulse) occurs for excitons in a semiconductor. In general the theory for this process in an ideal semiconductor is more complicated because of contributions from the continuum and can lead to more complex temporal 
structure in the time resolved emission [25-29]. However, in the presence of disorder, the extended state nature of the wave function is absent, and the data appears to be well described by the usual density matrix description of these systems, if appropriately generalized.

Coherent transient spectroscopy has been particularly useful in the study of disordered systems. A striking prediction due to interface disorder in two dimensional systems has been the presence of a mobility edge [30]. The mobility edge is the transition energy that divides localized states from extended states and is characterized by a corresponding increase in the exciton mobility (and diffusion coefficient) and dephasing rate. Strong evidence for this was first reported in [31] with similar behavior reported in hole-burning type experiments described above [19,32]. Indeed, the mobility edge is believed to occur near the mid-point of the inhomogeneous line, and in some cases hole burning was not observed above line center, indicating a fast dephasing time. However, the understanding of this behavior remains incomplete as not all structures are characterized by this edge. A model that relates the existence of the edge to a detailed description of the disorder is lacking in spite of excellent experiments characterizing these states (see for example [33] and theory and experiment in $[34,35])$.

More specifically, under experimental conditions that emphasize the presence of disorder $(T<5 \mathrm{~K}$ and low exciton density, $n<5 \times 10^{9} \mathrm{~cm}^{-2}$ ) the coherent transient response shows the complex nature of localization. For example, interpretation of the time integrated coherent FWM response for either homogeneously or inhomogeneously broadened systems that follow the theory for simple non-interacting twolevel systems is well understood (see for example [14]). However, in the presence of disorder, interpretation of the time integrated response can be considerably more complex. Measurements show that only at the lowest excitation levels is a simple photon echo observed in quantum well structures. At higher excitation levels, the time resolved emission shows the presence of two signals corresponding a free polarization decay as well as a photon echo [36], each signal arising from excitons characterized by different dephasing rates, exciton mobilities, etc, indicating the co-existence of both localized and extended states [37]. Such an interpretation was clearly controversial [38], but further theoretical support for such behavior has recently been presented [39] which shows that localized and delocalized states can exist at the same energy, though spatially distinct. Compelling data supporting this thinking is shown in Fig. 4 a which shows a time-integrated response of the FWM response [40]. In the usual picture, this response is a simple exponential decay, characteristic of the usual Markoff approximation. However, the decay in Fig. 4a is clearly multi-exponential. Moreover, the magnitude of the prefactors associated with the different exponential components depend on excitation energy. Assuming a simple bi-exponential and allowing the prefactors to vary with wavelength, the entire data set (the decay associated spectrum) is well fit without allowing the decay rates to be excitation energy dependent. Figure $4 \mathrm{~b}$ shows the magnitude of the two prefactors as a function of energy along with the corresponding exciton lineshape. The data clearly shows the presence of two classes of excitons characterized by different dephasing rates. This data, coupled with other measurements showing different activation energies for relaxation for each
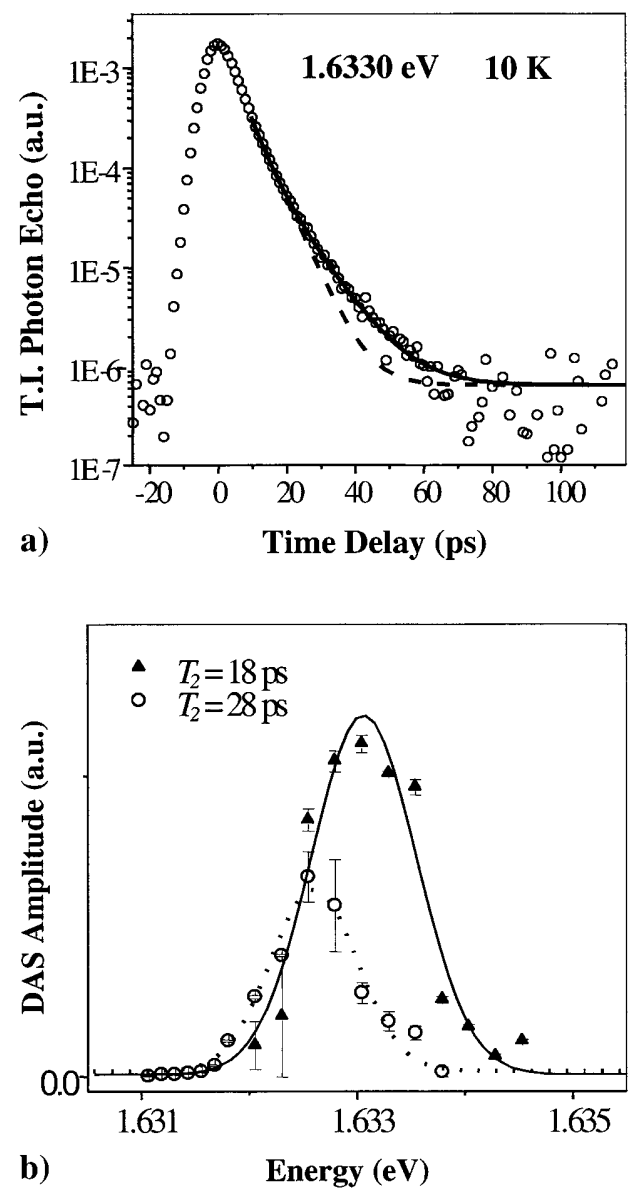

Fig. 4. a The time integrated photon echo decay measured near line center with a biexponential fit (full line) and a single exponential fit (dashed line). Also shown is the tripled laser pulse indicating the pulse-width limited part of the photon echo decay. b The decay associated spectra (DAS) corresponding to the fast (triangles) and slower (circles) component of the biexponential decay relative to the optical density of the resonance. The dashed line shows the absorption line shape

exciton component, demonstrates the theoretical prediction of the potential for both localized and extended states to coexist at the same energy, though in different regions of the sample. It is assumed in this interpretation that the faster dephasing rate of the higher energy states corresponds to exciton states with more extended wave functions. This assumption is supported by the fact that these states were also shown to have relatively high mobility compared to the lower energy states with smaller dephasing rates which had no detectable mobility, corresponding to strong localization.

Another distinct advantage of coherent transient nonlinear spectroscopy such as stimulated photon echoes is its ability to determine both the population decay, $\Gamma$, and the total dephasing rate, $\gamma$, thus enabling us to determine the pure dephasing contribution given by $\gamma_{\mathrm{ph}}=\gamma-\Gamma / 2$. While the concept of pure dephasing is well established in atomic physics, pure dephasing is not expected in ideal three-dimensional semiconductors because of crystal momentum conservation. For lower dimensional semiconductors, however, pure dephasing processes become important since the breaking of translation symmetry relaxes the requirement of crystal momentum conservation. In this regard, disorder in semiconductors can also strongly affect contributions of pure dephasing to the over- 
all dephasing process. In addition, the confinement in these structures can to a certain extent suppress energy or population relaxation.

A clear demonstration of pure dephasing in semiconductor heterostructures was carried out in a narrow GaAs/AlGaAs QW with a well width of $2.8 \mathrm{~nm}$ [41]. Lower energy excitons in these narrow QWs have been shown to be confined in interface islands with a lateral dimension of $40 \mathrm{~nm}$ [42-44]. The energy level structure of excitons also exhibits weak 3D confinement. Figure 5a shows experimental results that compare directly dephasing measurement with population decay measurement by using the stimulated photon echoes. Note that the population decay is characterized by two decay components. The fast component reflects overall population relaxation rate of excitons and includes contributions from exciton spectral diffusion, thermal activation to higher excited states as well as radiative recombination. The slower component is due to a thermalized exciton distribution with the relaxation rate determined by the exciton radiative recombination rate. Figure $5 \mathrm{~b}$ shows the corresponding energy dependence of the relaxation rates obtained at $10 \mathrm{~K}$. To avoid complications due to exciton-exciton interactions, data shown in Fig. 5b (and later in Fig. 5c) were obtained by extrapolating the decay rates to the zero exciton density limit. As shown in Fig. 5b, at very low temperature, $\gamma \sim \Gamma / 2$ and very little pure dephasing is observed. The absence of pure dephasing at very low temperature has also been confirmed by studies based on single exciton spectroscopy.

Pure dephasing contributions become important with increasing temperature and become the dominant contribution to the dephasing process at elevated temperatures as shown by the temperature dependence of both dephasing and population decay rates in Fig. 5c. The strong temperature dependence shows that the observed pure dephasing arises from exciton-phonon interactions. Pure dephasing induced by electron-phonon interactions can be understood qualitatively by using the Huang-Rhys model and can also be viewed as due to acoustic phonon side bands. Within the limit of the Huang-Rhys model, the primary effect of the excitonphonon coupling is a shift in the equilibrium position of lattice vibrations and a temperature-independent polaron shift of the exciton energy $[45,46]$. Thus, the ground and excited states of the coupled exciton-phonon system can be described (in terms of eigenfunctions of the uncoupled system) by $\left|\phi_{g}\right\rangle\left|\varphi_{m}(x)\right\rangle$ and $\left|\phi_{e}\right\rangle\left|\varphi_{n}(x-a)\right\rangle$, where $\varphi_{m}(x)$ is the wave function for a phonon state with $m$ phonons, $x$ is the phonon coordinate with a being a relative shift in the equilibrium position induced by the exciton-phonon coupling, and $\phi_{g}$ and $\phi_{e}$ are the wave functions of the electronic ground and excited states, respectively. The dipole matrix element between the ground and excited states of the coupled system is then $\left\langle\phi_{g}|\boldsymbol{r}| \phi_{e}\right\rangle\left\langle\varphi_{m}(x) \mid \varphi_{n}(x-a)\right\rangle$. The optical transition can take place between states involving different phonon numbers since $\left\langle\varphi_{m}(x) \mid \varphi_{n}(x-a)\right\rangle \neq 0$ even when $m \neq n$ and can be viewed as transitions between two quasi-continuous manifolds. The resulting spectral broadening of the optical transition depends on the relative strength of relevant transitions but does not involve population relaxation of the excitonic states.

To describe the pure dephasing processes in excitons with weak 3D confinement, it is also necessary to generalize the Huang-Rhys model to include off-diagonal electron-phonon coupling that mixes different excitonic states. The solid and
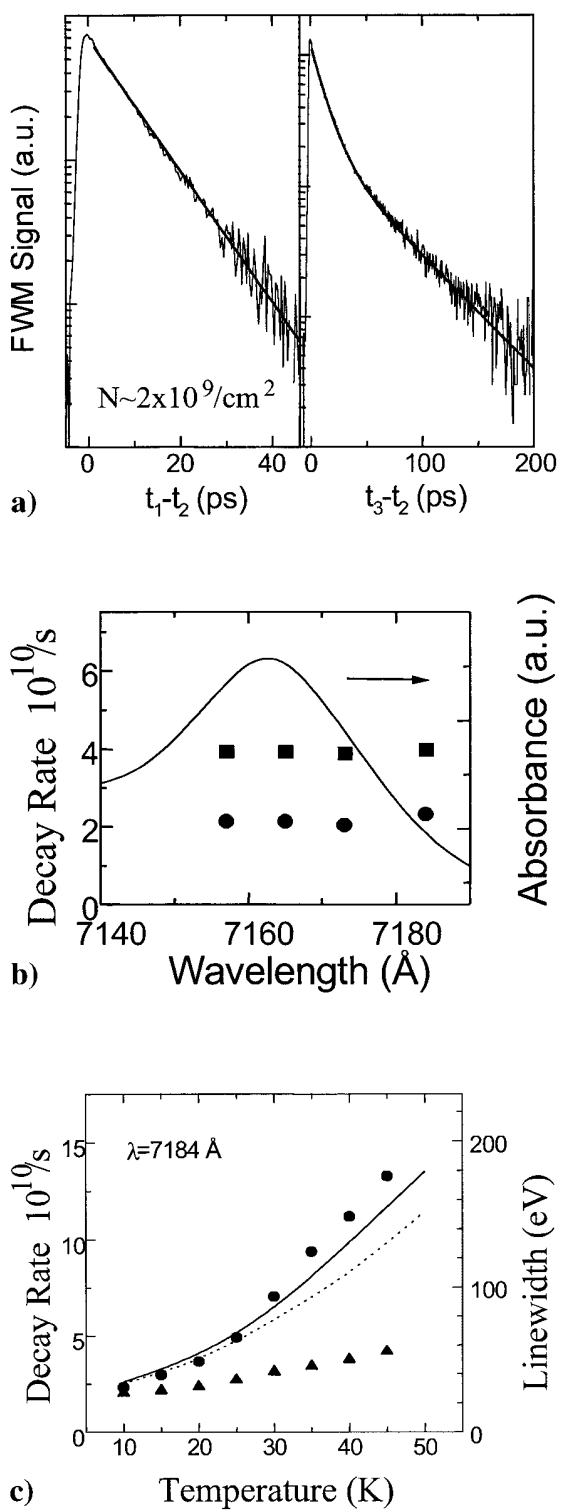

Fig. 5. a FWM response at $10 \mathrm{~K}$ for excitons at $7184 \AA$ A. Solid lines show the exponential fit to the response. (Right Panel) As a function of $t-t_{2}$ with $t_{1}=t_{3}$. Dephasing rates derived are $0.026 \mathrm{ps}^{-1}$. (Left Panel) As a function of $t_{3}-t_{2}$ with $t_{1}=t_{2}$. Population decay rates derived are $0.04 \mathrm{ps}^{-1}$ and $0.01 \mathrm{ps}^{-1}$ for the fast and slow component, respectively. b Energy dependence of exciton dephasing rates (circles) and population decay rates (squares) at $10 \mathrm{~K}$. Absorption spectrum of the lower energy hh exciton resonance is also plotted. c Dephasing rates (circles) and half population decay rates $\Gamma / 2$ (triangles) as a function of the temperature. The solid and dashed curves show theoretical results of dephasing rates for the exciton ground state with and without the contribution of off-diagonal electron-phonon interactions, respectively

dash lines in Fig. 5c shows theoretical calculations of dephasing rates with and without the contribution of off-diagonal electron-phonon coupling, respectively [41]. It should also be pointed out for semiconductor nanostructures with strong 3D confinement such as CdSe nanocrystals, contribution from the off-diagonal electron-phonon coupling to the pure dephasing process can become negligible due to large energy separation between relevant excitonic states.

Finally, we note that dephasing, while usually being considered a feature of the coherence decay rate reflected in 
either hole burning or coherent transient measurements, can also be a source of the coherent nonlinear optical response. This highly unintuitive result can be understood by considering the simple relationship between dephasing and exciton density. Namely, exciton induced dephasing (EID) is the result of the fact that the dephasing rate is given by the intrinsic dephasing rate, $\gamma$, and a contribution due to exciton scattering, $\xi N_{\mathrm{e}}$, where $\xi$ is a parameter related the scattering cross section, and $N_{\mathrm{e}}$ is the exciton density. The full polarization is given by $P=\kappa E \mathrm{e}^{-\mathrm{i} \omega t}\left[\omega-\omega_{0}-\mathrm{i}\left(\gamma_{0}+\xi N_{\mathrm{e}}\right)\right]^{-1}+c c$. Since $N_{\mathrm{e}}=\eta|E|^{2}$, a simple Taylor series expansion for weak fields provides a third order polarization proportional to $\xi$, the EID term. The initial evidence for the dominance of this term over other manybody contributions to coherent nonlinear optical response was quite striking as seen in a thin film of pure $\mathrm{GaAs}$ [47]. In a quantum well structure, the issues are more complicated as both local field effects [48] and the biexciton also play a role. But very sophisticated measurements of the time dependent E-field polarization reveal the deconvolution of these effects $[49,50]$.

In summary, we have reviewed the basic approaches to the study of excitonic dephasing in heterostructures, and examined some of the basic physical processes underlying the loss of quantum phase. The description remains necessarily qualitative as much remains to be understood in these systems including the development of a microscopic theory for disorder that is based on a more complete experimental understanding.

Acknowledgements. This work was supported by ARO, AFOSR, and the NSF Center for Ultrafast Optical Science.

\section{References}

1. T. Takagahara: Phys. Rev. B 32, 7013 (1985)

2. T. Takagahara: J. Lumin. 44, 347 (1989)

3. C. Lonsky, P. Thomas, A. Weller: Phys. Rev. Lett. 63, 652 (1989)

4. D. Brinkman, F. Rossi, S.W. Koch, P. Thomas: Phys. Rev. B 54, 2561 (1996)

5. D. Brinkmann, K. Bott, S.W. Koch, P. Thomas: Phys. Stat. Sol. 206, 493 (1998)

6. N.H. Bonadeo, D.G. Steel, R. Merlin: Phys. Rev. B 60, 8970 (1999)

7. H.F. Hess, E. Betzig, T.D. Harris, L.N. Pfeiffer, K.W. West: Science 264, 1740 (1994)

8. N.H. Bonadeo, G. Chen, D. Gammon, D.S. Katzer, D. Park, D.G. Steel: Phys. Rev. Lett. 81, 2759 (1998)

9. N.H. Bonadeo, A.S. Lenihan, G. Chen, J.R. Guest, D.G. Steel, D. Gammon, D.S. Katzer, D. Park: Appl. Phys. Lett. 75, 2933 (1999)

10. L. Schultheis, J. Kuhl, A, Honold, C.W. Tu: Phys. Rev. Lett. 57, 1635 (1986)

11. L. Schultheis, A. Honold, J. Kuhl, K. Kohler, C.W. Tu: Phys. Rev. B 34, 9027 (1986)

12. A. Honold, L. Schultheis, J. Kuhl, C.W. Tu: Phys. Rev. B 40, 6442 (1989)
13. L. Schultheis, J. Kuhl, A. Honold, C.W. Tu: Phys. Rev. Lett. 14, 1797 (1986)

14. A.M. Weiner, S. De Silvestri, E.P. Ippen: JOSA B 2, 654 (1985)

15. J. Shah: Ultrafast Processes in Semiconductors and Semiconductor Nanostructures (Springer, Berlin 1996)

16. D.G. Steel, J.T. Remillard: Phys. Rev. A 36, 4330 (1987)

17. H. Wang, D.G. Steel: Phys. Rev. A 43, 3823 (1991)

18. H. Wang, M. Jiang, R. Merlin, D.G. Steel: Phys. Rev. Lett. 69, 804 (1992)

19. H. Wang, M. Jiang, D.G. Steel: Phys. Rev. Lett. 65, 1255 (1990)

20. T. Tanaka, H. Sakaki: J. Cryst. Growth 81, 153 (1987)

21. J. Hegarty, K. Tai, W.T. Tsang: Phys. Rev. B 38, 7843 (1988)

22. N.A. Kurnit, I.D. Abella, S.R. Hartmann: PRL 13, 567 (1964)

23. T.W. Mossberg, A. Flusberg, R. Kachru, S.R. Hartmann: Phys. Rev. Lett. 42, 1665 (1979)

24. L. Schultheis, M.D. Sturge, J. Hegarty: Appl. Phys. Lett. 47, 995 (1985)

25. M. Wegener, D.S. Chemla, S. Schmitt-Rink, W. Schäfer: Phys. Rev. A 42, 5675 (1990)

26. M. Lindberg, R. Binder, S.W. Koch: Phys. Rev. A 45, 1865 (1992)

27. W. Schäfer, D.S. Kim, J. Shah, T.C. Damen, J.E. Cunningham, K.W. Goossen, L.N. Pfeiffer, K. Köhler: Phys. Rev. B 53, 16429 (1996)

28. D.S. Chemla: Semicond. Semimet. 58, 175 (1999)

29. K. Leo, E.O. Göbel, T.C. Damen, J. Shah, S. Schmitt-Rink, W. Schaefer, J.W. Müller, K. Köhler, P. Ganser: Phys. Rev. B 44, 5726 (1991)

30. N.F. Mott, E.A. Davis: Electronic Processes in Noncrystalline Materials, 2nd ed. (Oxford, Clarendon 1979)

31. J. Hegarty, L. Goldner, M.D. Sturge: Phys. Rev. B 30, 7346 (1984)

32. H. Wang, J.T. Remillard, M.D. Webb, D.G. Steel, J. Pamulapati, J. Oh, P.K. Bhattacharya: Surf. Sci. 228, 69 (1990)

33. R. Hellman, S.T. Cundiff, M. Koch, J. Feldmann, E.O. Göbel, B. Juhnheinrich, D.R. Yakovlev, A. Waag, G. Landwehr: Phys. Rev. B 50, 14651 (1994)

34. E.O. Göbel, P. Thomas: J. Non-Cryst. Solids 141, 35 (1992)

35. F. Jahnke, M. Koch, T. Meier, J. Feldmann, W. Schäfer, P. Thomas, S.W. Koch, E.O. Göbel, H. Nickel: Phys. Rev. B 50, 8114 (1994)

36. M.D. Webb, S.T. Cundiff, D.G. Steel: Phys. Rev. Lett. 66, 934 (1991)

37. S.T. Cundiff, H. Wang, D.G. Steel: Phys. Rev. B 46, 7248 (1992)

38. P.W. Anderson: Phys. Rev. 109, 1492 (1958)

39. U. Jahn, M. Ramsteiner, R. Hey, H.T. Grahn, E. Rnage, R. Zimmermann: Phys. Rev. B 56, R4387 (1997)

40. J. Erland, J.C. Kim, N.H. Bonadeo, D.G. Steel, D. Gammon, D. Katzer: Phys. Rev. B 60, R8497 (1999)

41. X. Fan, T. Takagahara, J.E. Cunningham, H. Wang: Solid State Commun. 108, 857 (1998)

42. D. Gammon, E.S. Snow, D.S. Katzer: Appl. Phys. Lett. 67, 2391 (1995)

43. D. Gammon, E.S. Snow, B.V. Shanabrook, D.S. Katzer, D. Park: Phys. Rev. Lett. 76, 3005 (1996)

44. D. Gammon, E.S. Snow, B.V. Shanabrook, D.S. Katzer, D. Park: Science 273, 87 (1996)

45. K. Huang, A. Rhys: Proc. R. Soc. London, Ser. A 204, 406 (1950)

46. C.B. Duke, G.D. Mahan: Phys. Rev. 139, A1965 (1965)

47. H. Wang, K. Ferrio, D.G. Steel, Y. Hu, R. Binder, S. Koch: Phys. Rev. Lett. 71, 1261 (1993)

48. K. Leo, M. Wegener, J. Shah, D.S. Chemla, E.O. Göbel, T.C. Damen, S. Schmitt-Rink, W. Schäfer: Phys. Rev. Lett. 65, 1340 (1990)

49. J.A. Bolger, A.E. Paul, A.L. Smirl: Phys. Rev. B 54, 11666 (1996)

50. A.L. Smirl, W.J. Walecki, X.Y. Chen, O. Buccafusca: Phys. Status Solidi B 204, 16 (1997) 